Original Article

\title{
Effect of ankle stretching combined with arm cycling on the improvement of calf muscle stiffness in patients with stroke: a pilot study
}

\author{
Akira OChI, RPT, MSc ${ }^{1,2)^{*}, \text { Masahisa Fukumoto, RPT }}{ }^{2)}$, Ryosuke TAKAmi, RPT ${ }^{3)}$, \\ Hiroshi Ohko, RPT, MSc ${ }^{1)}$, TAKahiro HaYashi, RPT, PhD ${ }^{1)}$, KaZumasa Yamada, RPT, PhD ${ }^{1,2)}$ \\ 1) Division of Physical Therapy, Faculty of Care and Rehabilitation, Seijoh University: 2-172 Fukinodai, \\ Toukai-City, Aichi 476-8588, Japan \\ 2) Department of Rehabilitation, Yamada Hospital, Japan \\ 3) Department of Rehabilitation, Geriatric Health Services Facility, Terada Garden, Japan
}

\begin{abstract}
Purpose] This study aimed to ascertain whether ankle stretching combined with arm cycling compared with that without arm cycling results in greater improvement in maximum dorsiflexion range of motion (ROM), calf muscle stiffness, and gait velocity in the affected limb of patients with stroke. [Participants and Methods] Random $\mathrm{ABAB}$ reversal design was used in this study involving nine patients with stroke. Participants performed $10 \mathrm{~min}$ of ankle plantar flexor stretching through weight bearing using the tilt table under the following 2 conditions: with or without arm cycling at 60 revolutions per minute. Pre- and post-stretching maximum ROM and passive plantar flexion torque were measured using a custom-made passive torque indicator. Stiffness was calculated based on passive torque-angle relationships. [Results] Maximum ROM and stiffness significantly improved after stretching in both conditions, whereas no changes in gait velocity were found. The difference in pre- and post-stretching stiffness was significantly greater in the stretching with arm cycling group. Change in maximum ROM showed no difference between both stretching conditions. [Conclusion] This study indicates that ankle plantar flexor stretching combined with arm cycling was more effective than that without cycling in improving calf muscle stiffness in the affected limb of patients with stroke.

Key words: Ankle stretching, Arm cycling, Stroke
\end{abstract}

(This article was submitted Jun. 8, 2018, and was accepted Jul. 26, 2018)

\section{INTRODUCTION}

Equinus foot due to spasticity of the lower limb after stroke onset affects standing up and walking independently ${ }^{1,2)}$. Ankle-foot orthosis is typically employed to manage equinus foot, which is necessary to achieve ambulation ${ }^{3)}$. However, a strong equinus deformity may arise from severe spasticity and/or joint contracture, which may cause back knee and difficulty in wearing a brace. Hence, ankle stretching through weight bearing using a tilt table ${ }^{4}$ is often performed from the early stage after stroke onset. Ankle plantarflexor stretching extends the maximum range of motion (ROM) during ankle dorsiflexion ${ }^{5-8)}$, decreases muscle-tendon unit stiffness due to passive torque reduction ${ }^{5-7,9)}$, and improves spasticity of the affected limb in patients with stroke as measured by modified Ashworth Scale (MAS) score ${ }^{8,9)}$. A previous study ${ }^{7)}$ reported that long-term stretching intervention for the plantarflexor muscle enhances the maximum voluntary contraction of the affected lower limb, resulting in an increased walking speed. However, in previous studies ${ }^{5-9)}$, a method of extending the ankle while controlling the resistance torque of the participant's hypertonia using an intelligent feedback-controlled device or a motor-driven device was employed, which is difficult to apply in the clinical setting. Constant torque stretch controlled with a motor-driven device

*Corresponding author. Akira Ochi (E-mail: ochi@seijoh-u.ac.jp)

(C2018 The Society of Physical Therapy Science. Published by IPEC Inc.

(c) (1) $\odot$ This is an open-access article distributed under the terms of the Creative Commons Attribution Non-Commercial No Deriva-

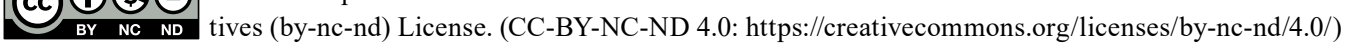


is more effective in reducing viscoelasticity of the spastic muscle than constant angle stretch ${ }^{8)}$. Nevertheless, constant angle stretching is often used in the clinical setting, as it effectively improves ROM and stiffness.

Moreover, previous studies showed that the H-reflex of the soleus muscle in the affected limb decreases during voluntary arm cycling in patients with stroke ${ }^{10}$. As H-reflex reflects motoneuron pool excitability in patients with stroke ${ }^{11)}$, H-reflex reduction may enhance the expansion effect of soft tissue extensibility by stretching. Voluntary arm cycling of the unaffected side in patients with stroke improves the spasticity of the affected upper limb based on MAS ${ }^{12)}$. However, some patients with stroke apparently have increased stretch reflex with voluntary arm cycling ${ }^{13)}$. Thus, other methods in which the participant could perform arm cycling with little physical effort, such as assisted motor arm cycling, have to be considered.

This pilot study aimed to ascertain whether ankle plantarflexor stretching using a tilt table combined with arm cycling with little physical effort improves maximum dorsiflexion ROM, calf muscle stiffness, and gait velocity in patients with stroke. It was hypothesized that stretching with arm cycling would result in greater ROM and more decreased stiffness in the affected limb of patients with stroke compared with the usual stretching with rested arm; the improvement would be reflected in the gait performance.

\section{PARTICIPANTS AND METHODS}

Nine patients ( 5 males; mean age $61.1 \pm 11.8$ years, body height $165.2 \pm 8.9 \mathrm{~cm}$, weight $63.0 \pm 8.5 \mathrm{~kg}$ ) who had stroke for the first time and were admitted to rehabilitation wards participated in this study. The inclusion criteria included ability to walk a 10-m distance independently without ankle-foot orthosis. The baseline characteristics of the participants are shown in Table 1. The exclusion criteria were as follows: severe ankle joint ROM limitations; inability to perform cycling movement with the upper limbs; and auditory or visual impairment. Those with consciousness disorder, cognitive impairment, or other severe cardiovascular diseases affecting exercise performance were also excluded. This study adhered to the Declaration of Human Rights, Helsinki, 1975, and was approved by the Ethical Review Board of Seijoh University (approval number: 2016A0029). Written informed consent was obtained from each participant.

Random ABAB reversal design was used in this study. Participants performed a 10-min ankle stretching using their own weight (non-use arm cycling [NAC], period A), and 10-min arm cycling was added to the stretching performed in period A (added arm cycling [AAC], period B). The exercise protocol was performed 2 days/week for 2 weeks, and the NAC and AAC conditions were provided randomly for each participant. All participants received routine rehabilitation, including muscular strengthening and balance, gait, and endurance training every day throughout the study period. The stretching was performed and measurements obtained after all the rehabilitation activities were completed.

An inclination plate for ankle dorsiflexion and a tilt table (GH-400, Og Giken Co. Ltd., Japan) were used for the static stretching. Participants leaned on the tilt table and stood with the knees extended on the inclination plate. Their chest and thighs were fixed with a belt. The inclination plate angle was determined based on the maximum dorsiflexion that the participants could achieve without insupportable pain in any joint and/or muscle. The inclination plate angle could be finely adjusted by placing a wood crosstie under the plate. The ankle dorsiflexion angle, which is related to the angle between the inclination plate and the tilt table, was $14.4 \pm 2.1^{\circ}$ in all participants. For the AAC condition, a portable cycle ergometer with a built-in servo motor (PBE-100, MEISEI Co. Ltd., Japan) was used. The cycle ergometer was placed on a height adjustable metal rack and was set according to the height of the sternum for the participants. The affected side of the upper limb was securely fastened to the pedal with a rubber belt. The participants were instructed to perform the arm cycling as voluntarily as possible with servo motor assistance at 60 revolutions per minute. The AAC condition is shown in Fig. 1.

Before and after the stretching in both the NAC and AAC conditions, the maximum ROM of ankle dorsiflexion and the passive plantarflexion torque of the affected limb were measured using the custom-made passive torque indicator (Takei Scientific Instruments Co. Ltd., Japan). The criterion-related validity of the stiffness measured with this equipment compared with that with isokinetic dynamometer (BIODEX ver.3) has been previously reported ${ }^{14}$ ). The custom-made passive torque indicator consisted of an angle-adjustable foot plate with strain gauge, digital angle gauge, and metal base frame, which allowed measurements of static plantarflexion torque and ankle dorsiflexion angle when the foot plate is moved to the passive dorsiflexion direction. Participants were in the supine position on a bed with the knee fully extended and the affected side of the foot was secured to the foot plate of the passive torque indicator. Passive torque was recorded when the value at the following dorsiflexion angles as the examiner moved the foot plate stabilized: $3,6,9$, and $12^{\circ}$. Moreover, the foot plate dorsiflexion angle where a slight pain in the calf was felt was regarded as the maximum ROM. The four points of the passive torque-angle relationships were plotted to create a scatter diagram, and a regression line fitted with least-squares method was drawn. The slope of the regression line was defined as stiffness $(\mathrm{Nm} / \mathrm{rad})$. The maximum ROM and stiffness measurements were performed three times, and the mean values were included in the data analysis. Before and after each stretching condition, mean walking speed at a 10-m distance and gait velocity obtained from a single trial of a 10-m walk were measured. Participants performed two walking trials at their maximum effort over a $12-\mathrm{m}$ walkway and the faster time for the middle $10 \mathrm{~m}$ was recorded.

PASW statistics ver. 18 (SPSS Inc., USA.) software was used for the statistical analysis. The normality of all distributions was verified using the Shapiro-Wilk test. The inter-day reliabilities for the pre-static stretching maximum ROM, stiffness, and gait velocity in four separate days were estimated using intra-class correlation coefficients (ICC). Comparisons of pre- and 
Table 1. Baseline characteristics of the participants

\begin{tabular}{|c|c|c|c|c|c|c|c|c|}
\hline ID & Gender & BMI & Stroke characteristics & $\begin{array}{c}\text { Time since } \\
\text { stroke (week) }\end{array}$ & Paretic side & BRS & $\mathrm{mRS}$ & MAS \\
\hline $\mathrm{a}$ & M & 22.6 & Ischemic & 10 & Rt & 4 & 3 & 1 \\
\hline $\mathrm{b}$ & $\mathrm{F}$ & 24.0 & Ischemic & 12 & $\mathrm{Lt}$ & 4 & 3 & $1+$ \\
\hline $\mathrm{c}$ & $\mathrm{F}$ & 25.1 & Ischemic & 11 & $\mathrm{Lt}$ & 4 & 3 & 1 \\
\hline $\mathrm{d}$ & M & 20.5 & Hemorrhagic & 6 & $\mathrm{Rt}$ & 4 & 2 & $1+$ \\
\hline $\mathrm{e}$ & $\mathrm{F}$ & 21.3 & Ischemic & 6 & $\mathrm{Lt}$ & 3 & 3 & 2 \\
\hline $\mathrm{f}$ & $\mathrm{F}$ & 22.1 & Ischemic & 11 & Rt & 3 & 3 & 2 \\
\hline $\mathrm{g}$ & M & 24.2 & Hemorrhagic & 9 & $\mathrm{Rt}$ & 3 & 3 & 2 \\
\hline $\mathrm{h}$ & M & 22.0 & Hemorrhagic & 5 & Rt & 4 & 2 & $1+$ \\
\hline $\mathrm{i}$ & M & 25.4 & Ischemic & 8 & $\mathrm{Lt}$ & 3 & 2 & 2 \\
\hline
\end{tabular}

M: male; F: female; BMI: body mass index; BRS: Brunnstrom recovery stage; mRS: modified Rankin Scale; MAS: modified Ashworth scale; Rt: right; Lt: left.

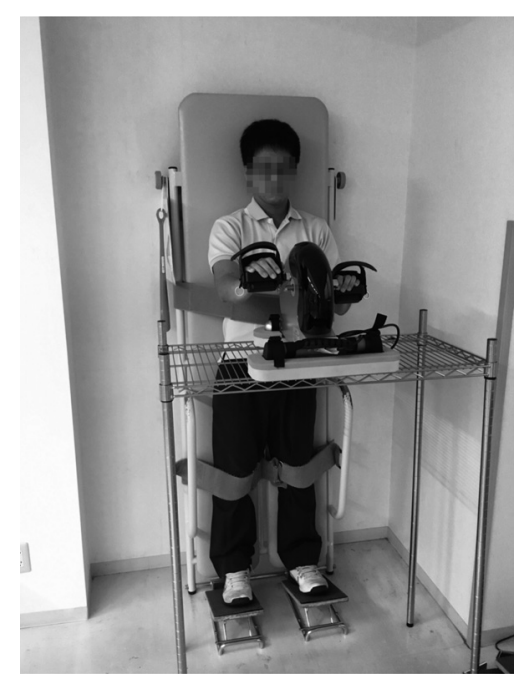

Fig. 1. Ankle stretching with added arm cycling (AAC) condition.

post-stretching under each condition was verified using a paired t-test. The rates of change in the three outcomes after NAC and AAC were calculated using the following formula: post - pre / pre $\times 100$ ), and the average values on days 1 and 2 were obtained. The average rate of changes in the maximum ROM, stiffness, and gait velocity (not following normal distribution) between the NAC and AAC was compared using the Wilcoxon signed-rank test. An effect size was confirmed when a significant difference in all statistical examinations was found. A p value $<0.05$ was considered statistically significant.

\section{RESULTS}

The ICCs (95\% confidence interval lower bound to upper bound) showing the inter-day reliabilities were 0.92 (0.77-0.98), 0.98 (0.93-0.99), and 0.99 (0.98-0.99) for the maximum ROM, stiffness, and gait velocity, respectively. These ICCs indicated almost perfect reliability. The pre-static stretching data and rate of changes in the four periods are provided in Table 2. For the pre- and post-static stretching comparison, the maximum ROM showed a significant increase after stretching in all periods: NAC day $1(\mathrm{t}=4.24, \mathrm{p}<0.01, \delta=0.65$; moderate), NAC day $2(\mathrm{t}=4.60, \mathrm{p}<0.01, \delta=0.70$; moderate), AAC day 1 ( $\mathrm{t}=5.87$, $\mathrm{p}<0.01, \delta=0.53$; moderate), and AAC day $2(\mathrm{t}=5.08, \mathrm{p}<0.01, \delta=0.62$; small). The stiffness also significantly decreased after stretching in all periods: NAC day $1(\mathrm{t}=3.47, \mathrm{p}<0.01, \delta=-0.20$; small $)$, NAC day $2(\mathrm{t}=3.48, \mathrm{p}<0.01, \delta=-0.21$; small $)$, AAC day $1(\mathrm{t}=6.83, \mathrm{p}<0.01, \delta=-0.49$; small), and AAC day $2(\mathrm{t}=4.21, \mathrm{p}<0.01, \delta=-0.65$; moderate). No significant difference in gait velocity in all conditions and periods was found. Only differences in stiffness between conditions were observed, and the rate of change in stiffness was significantly greater in $\mathrm{AAC}$ than in $\mathrm{NAC}(\mathrm{Z}=2.07, \mathrm{p}<0.05, \mathrm{r}=0.69$; large $)$. No significant difference in the rate of change in maximum ROM between AAC and NAC was observed. 
Table 2. Pre-stretching data and rate of changes (pre- and post-stretching) in the four periods

\begin{tabular}{lcccc}
\hline & NAC day 1 & NAC day 2 & AAC day 1 & AAC day 2 \\
\hline Pre ROM (deg) & $18.1 \pm 2.6$ & $17.6 \pm 3.1$ & $18.3 \pm 3.0$ & $17.7 \pm 4.0$ \\
Post - Pre \%change & $8.9 \pm 5.1 * *$ & $11.7 \pm 5.6 * *$ & $8.9 \pm 4.7 * *$ & $14.0 \pm 7.8 * *$ \\
Average value on days 1 and 2 (\%) & \multicolumn{2}{c}{$10.3 \pm 5.2$} & \multicolumn{2}{c}{$11.4 \pm 5.6$} \\
Pre Stiffness (Nm/rad) & $49.9 \pm 15.9$ & $49.1 \pm 15.4$ & $51.6 \pm 17.6$ & $49.0 \pm 12.6$ \\
Post - Pre \%change & $-8.0 \pm 8.0 * *$ & $-7.9 \pm 8.0 * *$ & $-20.7 \pm 7.5 * *$ & $-24.2 \pm 9.4 * *$ \\
Average value on days 1 and 2 (\%) & \multicolumn{2}{c}{$-7.9 \pm 8.1$} & \multicolumn{2}{c}{$-16.8 \pm 4.1 \dagger$} \\
Pre Gait velocity (m/s) & $0.73 \pm 0.39$ & $0.77 \pm 0.36$ & $0.77 \pm 0.35$ & $0.80 \pm 0.36$ \\
Post - Pre \%change & $5.9 \pm 8.8$ & $0.3 \pm 11.5$ & $1.2 \pm 3.6$ & $1.3 \pm 3.0$ \\
Average value on days 1 and 2 (\%) & \multicolumn{2}{c}{$3.1 \pm 7.7$} & \multicolumn{2}{c}{$1.2 \pm 2.6$} \\
\hline
\end{tabular}

Data were presented as mean \pm standard deviations. ROM: maximum range of motion for ankle dorsiflexion in the affected limb; NAC: non-use arm cycling condition; AAC: added arm cycling condition.

$* * \mathrm{p}<0.01$ significant difference for pre- vs. post-stretching.

$\uparrow \mathrm{p}<0.05$ significant difference for NAC vs. AAC (average value on days 1 and 2).

\section{DISCUSSION}

Static ankle stretching through weight bearing using a tilt table in both NAC and AAC conditions increased the maximum ankle dorsiflexion ROM and decreased the stiffness of spastic calf muscle in patients with stroke. The additional effect of AAC was reflected by the greater reduction in stiffness compared with that in NAC. However, the stretching intervention resulted in no significant change in gait velocity regardless of whether arm cycling was performed or not. These results supported one of our hypotheses, that is, stiffness reduction with AAC.

In this study, the calf muscle stiffness based on a custom-made passive torque indicator displayed a high inter-day reproducibility. Our previous work on healthy young adults ${ }^{14)}$ indicated that a fixed error between stiffness obtained from custom-made passive torque indicator and that from dynamometer exists. However, the validity of the stiffness between the two approaches is of no concern as this study is interested in the stiffness after the stretching intervention.

Muscle resistance due to tonic reflex activity is considered part of the limiting factor during stretching. Increased involuntary electromyographic activity of the corresponding muscle during static stretching is associated with passive resistance and prevents muscle extensibility ${ }^{15}, 16$. Adding arm cycling during ankle stretching is designed to achieve the superposition effect that inhibits motoneuron pool excitability in both calf stretch maneuver ${ }^{17}{ }^{18)}$ and arm cycling ${ }^{10,}{ }^{13}$, 19-22), thereby decreasing passive muscle resistance and expanding the maximum ROM. Thus, ankle stretching combined with arm cycling was considered the reason for the more decreased calf muscle stiffness in AAC. However, the rate of change in the maximum ROM was not significantly different between NAC and AAC. Maximum ROM was dependent on the changes in the factors involved in pain threshold, stretch tolerance, and involuntary activation of the agonist muscle ${ }^{23,24)}$. In this study, maximum ROM was determined based on the subjective pain during calf elongation, which in turn may have affected the links to stiffness reduction. Moreover, in this study, walking speed showed no changes after the intervention in both NAC and AAC conditions. Ankle stretching had no influence on walking kinematics ${ }^{25}$; nevertheless, a long-term stretching intervention may be necessary to achieve an increase in walking speed ${ }^{7)}$.

This study has several limitations. A previous study on the influence of various arm cycling parameters reported that the effect of motoneuron excitability suppression by arm cycling is promoted as the frequency (cadence) of cycling increases, while differences in crank road did not have a significant influence ${ }^{20}$. In addition, arm cycling must be a spontaneous drive, and reflectional suppression with external drive is associated with a limited effect ${ }^{20}$. In this study, AAC was used with the rotation speed kept constant; however, whether spontaneous upper limb muscle contraction continued for 10 min was not confirmed. Moreover, only a small number of stroke patients with mild to moderate spasticity were included in this study. Hence, whether individuals with more severe spasticity would benefit from arm cycling with stretching remains to be identified.

Ankle stretching through weight bearing with arm cycling could more effectively reduce calf muscle stiffness of the affected lower limb in patients with stroke compared with the usual stretching with rested upper limb. This stretching technique could be conveniently used in the clinical setting, has a low cost, and has clinical importance. Future research needs to investigate whether long-term stretching interventions using this stretching technique would result in long-term effects on ROM and stiffness.

\section{Funding}

This work was supported by Seijoh University Joint Research Promoting Grant. 


\section{Conflict of interest}

None of the authors have conflict of interest.

\section{REFERENCES}

1) Perry J, Garrett M, Gronley JK, et al.: Classification of walking handicap in the stroke population. Stroke, 1995, 26: 982-989. [Medline] [CrossRef]

2) Chen CL, Chen HC, Tang SF, et al.: Gait performance with compensatory adaptations in stroke patients with different degrees of motor recovery. Am J Phys Med Rehabil, 2003, 82: 925-935. [Medline] [CrossRef]

3) Chisholm AE, Perry SD: Ankle-foot orthotic management in neuromuscular disorders: recommendations for future research. Disabil Rehabil Assist Technol, 2012, 7: 437-449. [Medline] [CrossRef]

4) Tsai KH, Yeh CY, Chang HY, et al.: Effects of a single session of prolonged muscle stretch on spastic muscle of stroke patients. Proc Natl Sci Counc Repub China B, 2001, 25: 76-81. [Medline]

5) Chung S, Bai Z, Rymer WZ, et al.: Changes of reflex, non-reflex and torque generation properties of spastic ankle plantar flexors induced by intelligent stretching. Conf Proc IEEE Eng Med Biol Soc, 2005, 4: 3672-3675. [Medline]

6) Zhang LQ, Chung SG, Bai Z, et al.: Intelligent stretching of ankle joints with contracture/spasticity. IEEE Trans Neural Syst Rehabil Eng, 2002, 10: 149-157. [Medline] [CrossRef]

7) Selles RW, Li X, Lin F, et al.: Feedback-controlled and programmed stretching of the ankle plantarflexors and dorsiflexors in stroke: effects of a 4-week intervention program. Arch Phys Med Rehabil, 2005, 86: 2330-2336. [Medline] [CrossRef]

8) Yeh CY, Tsai KH, Chen JJ: Effects of prolonged muscle stretching with constant torque or constant angle on hypertonic calf muscles. Arch Phys Med Rehabil, 2005, 86: 235-241. [Medline] [CrossRef]

9) Yeh CY, Chen JJ, Tsai KH: Quantitative analysis of ankle hypertonia after prolonged stretch in subjects with stroke. J Neurosci Methods, 2004, 137: 305-314. [Medline] [CrossRef]

10) Barzi Y, Zehr EP: Rhythmic arm cycling suppresses hyperactive soleus H-reflex amplitude after stroke. Clin Neurophysiol, 2008, 119: 1443-1452. [Medline] [CrossRef]

11) Higashi T, Funase K, Kusano K, et al.: Motoneuron pool excitability of hemiplegic patients: assessing recovery stages by using H-reflex and M response. Arch Phys Med Rehabil, 2001, 82: 1604-1610. [Medline] [CrossRef]

12) Sakamoto K, Nakamura T, Uenishi H, et al.: Immediate effects of unaffected arm exercise in poststroke patients with spastic upper limb hemiparesis. Cerebrovasc Dis, 2014, 37: 123-127. [Medline] [CrossRef]

13) Mezzarane RA, Nakajima T, Zehr EP: After stroke bidirectional modulation of soleus stretch reflex amplitude emerges during rhythmic arm cycling. Front Hum Neurosci, 2014, 8: 136. [Medline] [CrossRef]

14) Ochi A, Fukumoto M, Takami R, et al.: Criterion-related validity and reliability of the surae muscle tendon unit stiffness measured using the custom made passive torque indicator. Rigakuryoho Kagaku, 2018, 33: 591-596 (In Japanese).

15) Moore MA, Hutton RS: Electromyographic investigation of muscle stretching techniques. Med Sci Sports Exerc, 1980, 12: 322-329. [Medline] [CrossRef]

16) Gajdosik RL: Influence of a low-level contractile response from the soleus, gastrocnemius and tibialis anterior muscles on viscoelastic stress-relaxation of aged human calf muscle-tendon units. Eur J Appl Physiol, 2006, 96: 379-388. [Medline] [CrossRef]

17) Guissard N, Duchateau J, Hainaut K: Muscle stretching and motoneuron excitability. Eur J Appl Physiol Occup Physiol, 1988, 58: 47-52. [Medline] [CrossRef]

18) Guissard N, Duchateau J, Hainaut K: Mechanisms of decreased motoneurone excitation during passive muscle stretching. Exp Brain Res, 2001, 137: 163-169. [Medline] [CrossRef]

19) Frigon A, Collins DF, Zehr EP: Effect of rhythmic arm movement on reflexes in the legs: modulation of soleus H-reflexes and somatosensory conditioning. J Neurophysiol, 2004, 91: 1516-1523. [Medline] [CrossRef]

20) Hundza SR, de Ruiter GC, Klimstra M, et al.: Effect of afferent feedback and central motor commands on soleus H-reflex suppression during arm cycling. J Neurophysiol, 2012, 108: 3049-3058. [Medline] [CrossRef]

21) Klarner T, Barss TS, Sun Y, et al.: Long-term plasticity in reflex excitability induced by five weeks of arm and leg cycling training after stroke. Brain Sci, 2016, 6: 54. [Medline] [CrossRef]

22) Zehr EP, Loadman PM, Hundza SR: Neural control of rhythmic arm cycling after stroke. J Neurophysiol, 2012, 108: 891-905. [Medline] [CrossRef]

23) Marsh E, Sale D, McComas AJ, et al.: Influence of joint position on ankle dorsiflexion in humans. J Appl Physiol, 1981, 51: 160-167. [Medline] [CrossRef]

24) McHugh MP, Kremenic IJ, Fox MB, et al.: The role of mechanical and neural restraints to joint range of motion during passive stretch. Med Sci Sports Exerc, 1998, 30: 928-932. [Medline]

25) Maynard V, Bakheit AM, Shaw S: Comparison of the impact of a single session of isokinetic or isotonic muscle stretch on gait in patients with spastic hemiparesis. Clin Rehabil, 2005, 19: 146-154. [Medline] [CrossRef] 